\title{
Different influence of polyoxygenation on the catalytic activity of amido- versus amino-isoborneols
}

\author{
Esther M. Sánchez-Carnerero, Tomás de las Casas Engel, Beatriz Lora Maroto, and \\ Santiago de la Moya Cerero*
}

Departamento de Química Orgánica I, Facultad de Ciencias Químicas, Universidad Complutense de Madrid, Avenida Complutense s/n, Madrid, 28040 Spain

E-mail: santmoya@quim.ucm.es

\begin{abstract}
A new series of polyoxygenated amido-isoborneols has been synthesized and tested in the enantioselective addition of diethylzinc to benzaldehyde and the results compared to a series of related amino isoborneols. The observed different behavior is explained on the basis of catalytic chelates previously proposed by us.
\end{abstract}

Keywords: Asymmetric catalysis, zinc, hydroxyamides, amino-isoborneols, structure-activity relationships

\section{Introduction}

One of the most synthetically useful methods to create carbon-carbon bonds in an asymmetric way is the enantioselective addition of organozinc reagents to aldehydes. ${ }^{1-12}$ Additionally, the reaction leads to secondary alcohols, which are valuable chiral building blocks ${ }^{13,14}$ for the preparation of natural products or new materials with interesting physicochemical properties, ${ }^{2,9}$ such as chiral liquid crystals ${ }^{15}$ (Scheme 1 ).

Although many chiral ligands for the reaction have been described so far, most of them being $N, N$-dialkyl amino alcohols (e.g. Noyori's DAIB 1, ${ }^{16}$ Pericàs' ephedrine analogue $2^{17}$ or Wang's ferrocene amino alcohol 3, ${ }^{18}$ Figure 1), there is still a search for a very versatile ligand, able to promote the addition of a wide range of organozinc reagents to a wide range of substrates. Moreover, the syntheses leading to the most efficient ligands are frequently long and resolution or asymmetric synthesis steps are often required in their preparation. 


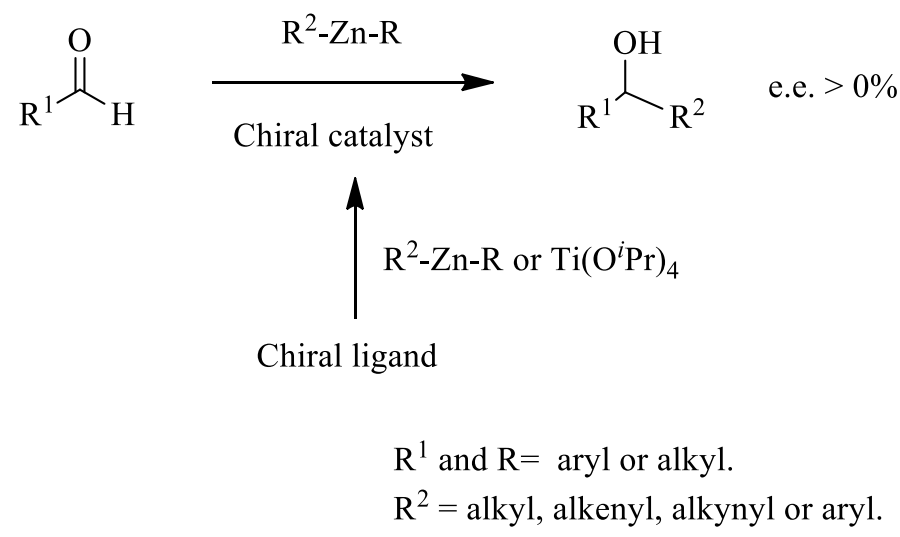

Scheme 1. Enantioselective addition of organozinc reagents to aldehydes.

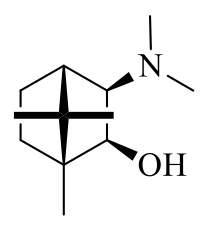

1

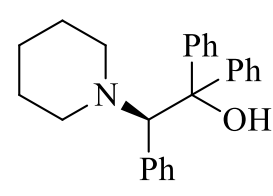

2

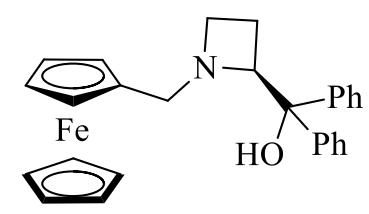

3

Figure 1. Some efficient ligands for the addition of organozinc reagents to aldehydes.

In the search for new versatile ligands, consideration of how the structural features of the ligands affect their catalytic activity is necessary. However, only a few studies on this topic have been carried out up to now. In this sense, we have reported a study on the effect of polyoxygenation on the catalytic activity of a series of amino isoborneols 4a-c (Figure 2). ${ }^{19} \mathrm{We}$ found a switch in the enantioselection of the ligands based on the polyoxygenation. We explained this switch by competition by a new transition state 6 (Figure 3), where the two additional oxygen atoms in the ligand act as a pincer, directing the diethylzinc molecule to the $s i$ face of benzaldehyde.

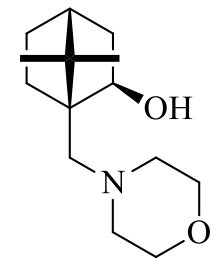

$4 \mathbf{a}$

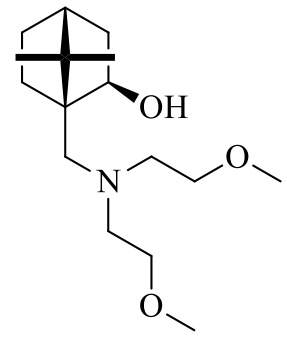

4b

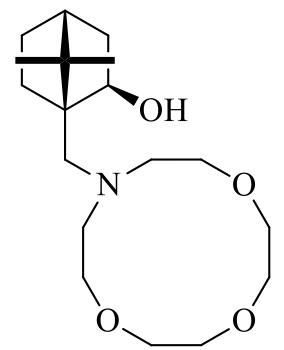

$4 c$

Figure 2. Polyoxygenated amino isoborneols. 


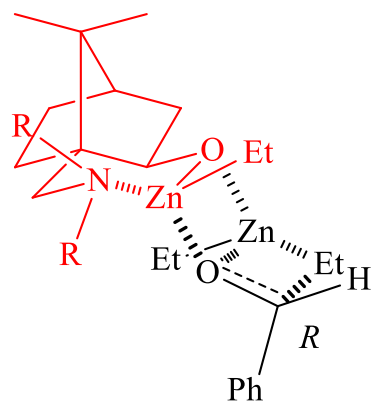

5

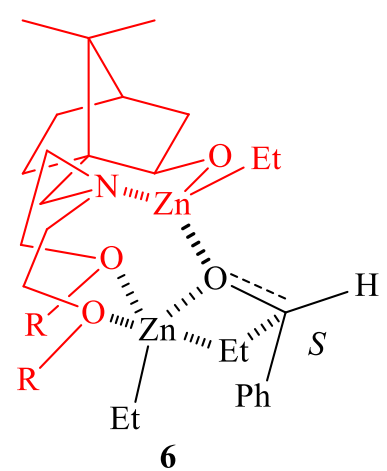

6

Figure 3. Proposed competitive TSs for amino isoborneols: 5 and 6 (6 is only possible for polyoxygenated amino isoborneols). (Catalytic Zn-chelate in red).

Additionally, we have found that some amido isoborneols have a catalytic activity comparable to related amino isoborneols and, thus, we have described ketopinic acid-derived $C_{2^{-}}$ symmetric bis(hydroxyamides) as efficient ligands to promote the enantioselective addition of diethylzinc to aldehydes. ${ }^{20,21}$ The advantage of the ligands having an amido group is that they are easily prepared and much more inert and stable than their analogous amines. In principle, the activity of these ligands could also be modulated by polyoxygenation of their amide moieties, as was the case for related amino isoborneols, ${ }^{19}$ however the effect of polyoxygenation in amido isoborneols has not been studied yet.

\section{Results and Discussion}

Following our investigations, we were interested in studying the effect that polyoxygenation has on the catalytic activity of amido isoborneols. To this end, we synthesized ligands 7a-c, having different numbers of oxygen atoms in their structures (Figure 4). These ligands were obtained in two straightforward steps from (1S)-ketopinic acid $\mathbf{8}$, a commercially available derivative of the naturally occurring camphor (Chiral Pool). The first step was the amidation of the acid with the corresponding oxygenated amine, promoted by EDC/DMAP, which afforded the intermediate keto amides 9 in good yields. ${ }^{19,22}$ Next, a chemo- and stereoselective reduction of the ketone group with $\mathrm{NaBH}_{4}$ gave the exo-hydroxy derivative 7 (isoborneol derivative) in good yields and very high stereoselectivity ${ }^{23}$ (Scheme 2 ). 


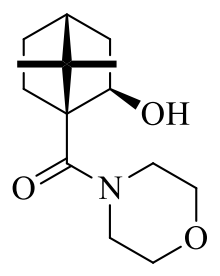

$7 \mathbf{a}$

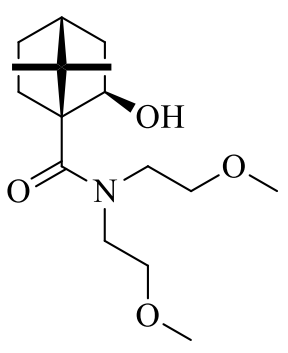

$7 \mathbf{b}$

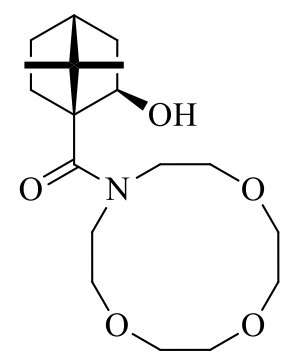

$7 \mathrm{c}$

Figure 4. Polyoxygenated amido isoborneols.

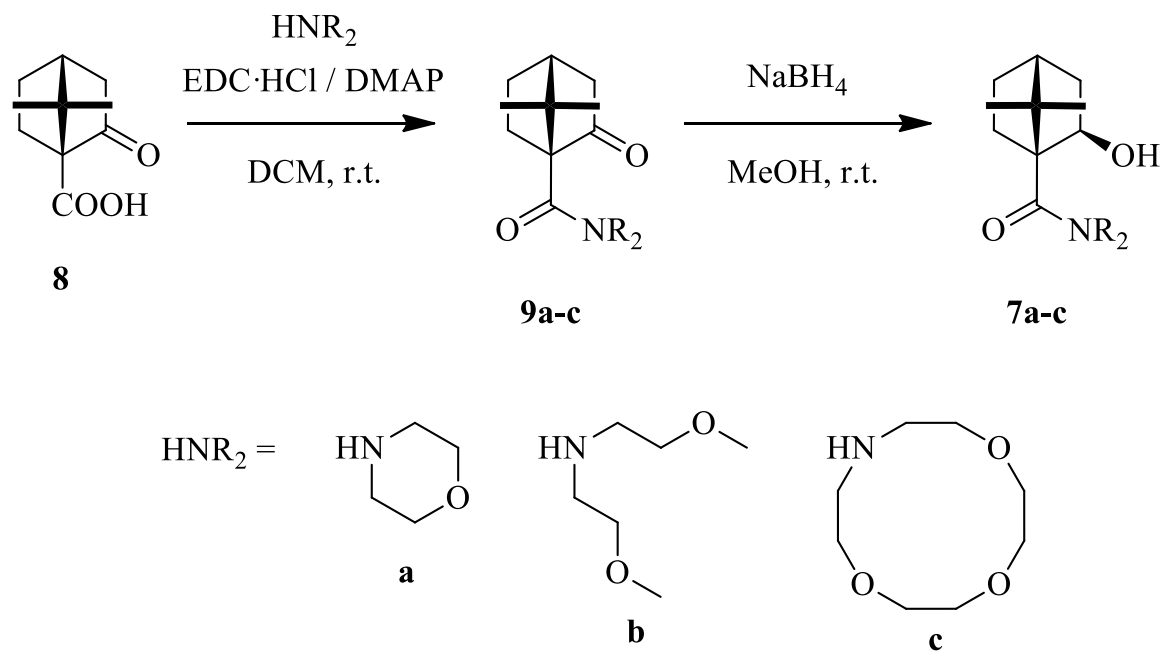

Scheme 2. Synthesis of polyoxygenated amido isoborneols 7 starting from commercial ketopinic acid 8.

The catalytic activity of ligands $7 \mathbf{a}-\mathbf{c}$ was evaluated in the enantioselective addition of diethylzinc to benzaldehyde. The results are shown in Table 1. The catalytic activity of ligands 4a-c has been included for comparison. ${ }^{19}$

The data from Table 1 show that there is no change in the catalytic activity of amido isoborneols 7a-c as a consequence of the different number of oxygen atoms present in the structure of the ligand. In contrast, the related amino alcohols $4 \mathbf{a}-\mathbf{c}$ did show different behavior, and even a reversal in the enantioselectivity, depending on the polyoxygenation. ${ }^{19}$ This different behavior of amino and amido isoborneols can be explained on the basis of the empirical catalyst models used previously by us $\mathbf{1 0}$ and $\mathbf{1 1}$ (Figure 5), hence corroborating our hypothesis. ${ }^{20}$ Thus, for amino isoborneols, the groups attached to the nitrogen that coordinates the catalytic zinc are near the active site of the catalyst $\mathbf{1 0}$. Therefore, they can influence the catalytic activity, as was actually observed. ${ }^{19}$ However, in amido isoborneols, the catalytic zinc is coordinated by the 
carbonyl oxygen (catalyst 11), which places the alkyl groups far away from the catalytic site. Hence, they cannot affect the activity of the catalyst.

Table 1. Catalytic activity of ligands 4 and 7 on the addition of diethylzinc to benzaldehyde

\begin{tabular}{ccccc}
\hline \multirow{2}{*}{ Entry } & \multirow{2}{*}{ Ligand } & \multicolumn{3}{c}{ 1-Phenylpropan-1-ol } \\
\cline { 3 - 5 } & & ${\text { Yield }(\%)^{\mathrm{b}}}^{\mathbf{c}}$ & $e e(\%)^{\mathrm{c}}$ & Configuration $^{\mathrm{d}}$ \\
\hline 1 & $\mathbf{4 a}^{19}$ & 99 & 72 & $R$ \\
2 & $\mathbf{4 b}^{19}$ & 99 & 36 & $S$ \\
3 & $\mathbf{4 c}^{19}$ & 99 & 52 & $R$ \\
4 & $\mathbf{7 a}$ & 94 & 82 & $R$ \\
5 & $\mathbf{7 b}$ & 98 & 78 & $R$ \\
6 & $\mathbf{7 c}$ & 98 & 78 & $R$ \\
\hline
\end{tabular}

${ }^{a}$ Benzaldehyde/diethylzinc/ligand: 1/2/0.05. $1 \mathrm{~mL}$ additional hexane, 5 h, r.t. ${ }^{\mathrm{b}}$ Determined by GC. ' Determined by chiral HPLC (Chiralpak IC). ${ }^{\mathrm{d}}$ Determined by chiral HPLC, by comparison with a known sample.

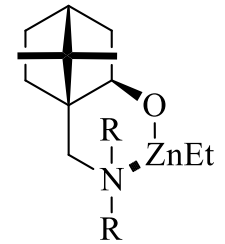

10

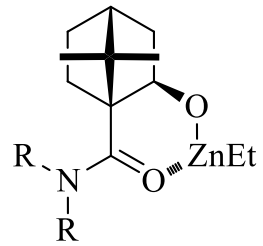

11

$\mathrm{R}=$ alkyl group, functionalized with coordinative heteroatom(s) in 2-position.

Figure 5. Proposed catalysts for amino isoborneols $\mathbf{1 0}$ and amido isoborneols $\mathbf{1 1}$.

\section{Conclusions}

The different influence of polyoxygenation in the catalytic activity of amido and amino isoborneols on the enantioselective addition of diethylzinc to benzaldehyde corroborates the different zinc chelate models proposed for each type of ligand and leads us to conclude that, unlike amino isoborneols, it is not possible to modulate the catalytic activity of amido isoborneols by introducing oxygen atoms in the amine moiety.

\section{Experimental Section}

General. Common solvents were dried and distilled by standard procedures. All starting materials and reagents were obtained commercially and used without further purification. Flash 
chromatography purifications were performed on silica gel (230-400 mesh ASTM). Melting points are uncorrected. NMR spectra were recorded at $20{ }^{\circ} \mathrm{C}$ in $\mathrm{CDCl}_{3}$ and the residual solvent peak was used as internal standard. FTIR spectra were obtained using the thin-films. GC analyses were realized at $120{ }^{\circ} \mathrm{C}$ in a chromatograph equipped with a capillary silicon-gum (SGL-1) column and a FID, and using nitrogen as mobile phase. Chiral-HPLC analyses were realized at r.t. in a chromatograph equipped with a Chiralpak-IC column and a DAD, and using hexane/isopropanol (98:2) as mobile phase. MS were recorded using the ESI or ESI-negative mode ionization techniques. HRMS were realized using FTMS (ESI or ESI-negative mode).

Ligand (7a). (1S,2R)-7,7-Dimethyl-1-[(morpholin-4-yl)carbonyl]norbornan-2-ol (7a). A twonecked round-bottom flask, equipped with a magnetic stirrer was charged with (1S)-7,7dimethyl-1-[(morpholin-4-yl)carbonyl]norbornan-2-one 9a ${ }^{22}$ (150 mg, $\left.0.6 \mathrm{mmol}\right)$, methanol (10 $\mathrm{mL})$ and $\mathrm{NaBH}_{4}(91 \mathrm{mg}, 2.4 \mathrm{mmol})$ under argon. The mixture was stirred for $24 \mathrm{~h}$. Then, water $(0.5 \mathrm{~mL})$ was added and the resulting mixture was concentrated under reduced pressure to evaporate methanol. The obtained residue was dissolved in $\mathrm{CHCl}_{3}(5 \mathrm{~mL})$ then, water $(3 \mathrm{~mL})$ was added, and the resulting layers were separated. The aqueous layer was extracted with chloroform ( $3 \times 3 \mathrm{~mL})$. The combined organic extracts were washed with brine $(1 \times 5 \mathrm{~mL})$ and dried over anhydrous $\mathrm{MgSO}_{4}$. After filtration and solvent evaporation under reduced pressure, the residue was purified by flash column chromatography ( silica gel, $\mathrm{CHCl}_{3}$ ).

7a. White solid, yield 88\%, $133 \mathrm{mg}$, mp 163-165 ${ }^{\circ} \mathrm{C}$. $[\alpha]_{\mathrm{D}}{ }^{20}-10.1$ (c 0.975, $\left.\mathrm{CHCl}_{3}\right) .{ }^{1} \mathrm{H} \mathrm{NMR}$ $\left(\mathrm{CDCl}_{3}, 300 \mathrm{MHz}\right), \delta: 4.16(\mathrm{dd}, J 7.9,4.3 \mathrm{~Hz}, 1 \mathrm{H}), 3.75-3.64(\mathrm{~m}, 8 \mathrm{H}), 2.06-1.75(\mathrm{~m}, 5 \mathrm{H}), 1.65$ $(\mathrm{dd}, J 4.3,4.3 \mathrm{~Hz}, 1 \mathrm{H}), 1.53-1.45(\mathrm{~m}, 1 \mathrm{H}), 1.38(\mathrm{~s}, 3 \mathrm{H}), 1.19-1.11(\mathrm{~m}, 1 \mathrm{H}), 1.15(\mathrm{~s}, 3 \mathrm{H}) \mathrm{ppm} .{ }^{13} \mathrm{C}$ NMR $\left(\mathrm{CDCl}_{3}, 75 \mathrm{MHz}\right), \delta: 171.9(\mathrm{C}), 78.0(\mathrm{CH}), 67.0\left(\mathrm{CH}_{2}\right), 60.6(\mathrm{C}), 50.6(\mathrm{C}), 44.81\left(\mathrm{CH}_{2}\right)$, $44.76(\mathrm{CH}), 41.5\left(\mathrm{CH}_{2}\right), 29.9\left(\mathrm{CH}_{2}\right), 27.0\left(\mathrm{CH}_{2}\right), 22.1\left(\mathrm{CH}_{3}\right), 21.6\left(\mathrm{CH}_{3}\right)$ ppm. FTIR, v $\max : 3508$ (w), 3376 (broad, w), 1738 (str), $1117(\mathrm{~m}) \mathrm{cm}^{-1}$. MS (ESI-negative mode) $\mathrm{m} / z$ (\%): 252 (100). HRMS (FTMS-ESI-negative mode) m/z: 252.1600 (calcd. for $\mathrm{C}_{14} \mathrm{H}_{22} \mathrm{NO}_{3}$ : 252.1605).

Ligand (7b). (1S)-1-\{[Bis(2-methoxyethyl)amino]carbonyl\}-7,7-dimethylnorbornan-2-one (9b). To a solution of (1S)-ketopinic acid 8 (291 mg, $1.6 \mathrm{mmol})$ in $\mathrm{CH}_{2} \mathrm{Cl}_{2}(5 \mathrm{~mL})$ at r.t. were added $N$-[3-(dimethylamino)propyl]- $N^{\prime}$-ethylcarbodiimide hydrochloride (EDC. $\mathrm{HCl}, 346 \mathrm{mg}$, $1.8 \mathrm{mmol}$ ), 4-(dimethylamino)pyridine (DMAP, $220 \mathrm{mg}, \quad 1.8 \mathrm{mmol}$ ) and bis(2methoxyethyl)amine $(239 \mathrm{mg}, 1.8 \mathrm{mmol}$ ). The reaction mixture was stirred for $24 \mathrm{~h}$. Then, $\mathrm{CHCl}_{3}(5 \mathrm{~mL})$ and water $(5 \mathrm{~mL})$ were added and the phases separated. The organic phase was washed successively with $10 \% \mathrm{HCl}(1 \times 3 \mathrm{~mL})$, water $(1 \times 3 \mathrm{~mL}), 10 \% \mathrm{NaOH}(2 \times 3 \mathrm{~mL})$, water (1 $\mathrm{x} 3 \mathrm{~mL}$ ) and brine (1 x $3 \mathrm{~mL}$ ), and dried over $\mathrm{MgSO}_{4}$. After filtration, the solvent was evaporated under reduced pressure giving $\mathbf{9 b}$ which was used for the next step without further purification.

9b. White solid, yield 77\%, $366 \mathrm{mg}$. $[\alpha]_{\mathrm{D}}{ }^{20}-24.5\left(c\right.$ 0.55, $\left.\mathrm{CHCl}_{3}\right) .{ }^{1} \mathrm{H} \mathrm{NMR}\left(\mathrm{CDCl}_{3}, 300 \mathrm{MHz}\right)$, $\delta$ : 3.70-3.31 (m, 8H), $3.31(\mathrm{~s}, 6 \mathrm{H}), 2.46(\mathrm{ddd}, J 18.4,4.7,2.6 \mathrm{~Hz}, 1 \mathrm{H}), 2.33-2.19(\mathrm{~m}, 1 \mathrm{H}), 2.16-$ $1.91(\mathrm{~m}, 3 \mathrm{H}), 1.88(\mathrm{~d}, J 18.4 \mathrm{~Hz}, 1 \mathrm{H}), 1.47-1.36(\mathrm{~m}, 1 \mathrm{H}), 1.17(\mathrm{~s}, 3 \mathrm{H}), 1.16(\mathrm{~s}, 3 \mathrm{H}) \mathrm{ppm} .{ }^{13} \mathrm{C}$ 
NMR $\left(\mathrm{CDCl}_{3}, 75 \mathrm{MHz}\right), \delta$ : $212.9(\mathrm{C}), 169.9(\mathrm{C}), 72.0\left(\mathrm{CH}_{2}\right), 70.8\left(\mathrm{CH}_{2}\right), 68.1(\mathrm{C}), 59.2\left(\mathrm{CH}_{3}\right)$, $51.1(\mathrm{C}), 48.7\left(\mathrm{CH}_{2}\right), 47.1\left(\mathrm{CH}_{2}\right), 44.6\left(\mathrm{CH}_{2}\right), 43.5(\mathrm{CH}), 28.2\left(\mathrm{CH}_{2}\right), 27.4\left(\mathrm{CH}_{2}\right), 21.8\left(\mathrm{CH}_{3}\right)$, $21.2\left(\mathrm{CH}_{3}\right)$ ppm. FTIR, v $v_{\max }: 1741$ (str), 1632 (str), 1119 (str) $\mathrm{cm}^{-1}$. MS (ESI) $\mathrm{m} / z$ (\%): 320 (100), 298 (1). HRMS (FTMS-ESI) m/z: 298.2026 (Calcd. for $\mathrm{C}_{16} \mathrm{H}_{28} \mathrm{NO}_{4}$ : 298.2013). (1S,2R)-1-

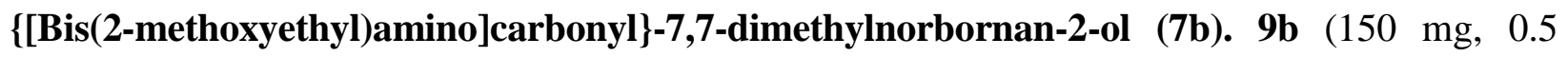
mmol) was reduced following the experimental procedure for the preparation of 7a. Purification was by column chromatography in hexanes /ethyl acetate (1/1).

7b. Colorless oil, yield 97\%, $147 \mathrm{mg}$. $[\alpha]_{\mathrm{D}}{ }^{20}+12.3\left(c\right.$ 0.81, $\left.\mathrm{CHCl}_{3}\right) .{ }^{1} \mathrm{H} \mathrm{NMR}\left(\mathrm{CDCl}_{3}, 300\right.$ $\mathrm{MHz}), \delta: 4.40$ (br s, 1H), $4.13(\mathrm{~m}, 1 \mathrm{H}), 3.67-3.28$ (m, 8H), 3.32 (s, 6H), 1.94-1.85 (m, 2H), 1.82$1.71(\mathrm{~m}, 2 \mathrm{H}), 1.54$ (dd, J 4.2, $4.2 \mathrm{~Hz}, 1 \mathrm{H}), 1.50-1.45(\mathrm{~m}, 1 \mathrm{H}), 1.34$ (s, 3H), 1.15-1.06 (m, 1H), $1.11(\mathrm{~s}, 3 \mathrm{H}) \mathrm{ppm} .{ }^{13} \mathrm{C} \mathrm{NMR}\left(\mathrm{CDCl}_{3}, 75 \mathrm{MHz}\right), \delta: 173.1(\mathrm{C}), 76.9(\mathrm{CH}), 70.8\left(\mathrm{CH}_{2}\right), 69.5\left(\mathrm{CH}_{2}\right)$, $61.2(\mathrm{C}), 58.8\left(\mathrm{CH}_{3}\right), 50.4(\mathrm{C}), 47.5\left(\mathrm{CH}_{2}\right), 45.5\left(\mathrm{CH}_{2}\right), 44.6(\mathrm{CH}), 39.7\left(\mathrm{CH}_{2}\right), 29.6\left(\mathrm{CH}_{2}\right), 27.2$ $\left(\mathrm{CH}_{2}\right), 22.3\left(\mathrm{CH}_{3}\right), 21.1\left(\mathrm{CH}_{3}\right)$ ppm. FTIR, v $v_{\max }: 3446$ (broad, w), $1628(\mathrm{str}), 1115(\mathrm{~m}) \mathrm{cm}^{-1} . \mathrm{MS}$ (ESI) $m / z \quad(\%): 621$ (100), 322 (73). HRMS (FTMS-ESI) m/z: 322.1979 (calcd. for $\mathrm{C}_{16} \mathrm{H}_{29} \mathrm{NNaO}_{4}$ : 322.1989).

Ligand (7c). (1S)-7,7-Dimethyl-1-[(1,4,7-trioxa-10-azacyclododecan-10-yl)carbonyl]norbornan-2-one (9c). (1S)-ketopinic acid (293 mg, $1.6 \mathrm{mmol}$ ) was reacted with 1,4,7-trioxa10-azacyclododecane, instead of bis(2-methoxyethyl)amine, following the experimental procedure for the preparation of $\mathbf{9 b}$.

9c. White solid, yield $83 \%, 453 \mathrm{mg}, \mathrm{mp} 85-87{ }^{\circ} \mathrm{C}$. $[\alpha]_{\mathrm{D}}{ }^{20}-19.3\left(c 0.460, \mathrm{CHCl}_{3}\right)$. ${ }^{1} \mathrm{H}$ NMR $\left(\mathrm{CDCl}_{3}, 300 \mathrm{MHz}\right), \delta: 3.99-3.24(\mathrm{~m}, 16 \mathrm{H}), 2.48$ (ddd, $J$ 18.4, 4.4, $\left.2.7 \mathrm{~Hz}, 1 \mathrm{H}\right), 2.35-1.93$ (m, $4 \mathrm{H}), 1.89(\mathrm{~d}, J 18.4 \mathrm{~Hz}, 1 \mathrm{H}), 1.48-1.37(\mathrm{~m}, 1 \mathrm{H}), 1.20(\mathrm{~s}, 3 \mathrm{H}), 1.18(\mathrm{~s}, 3 \mathrm{H}) \mathrm{ppm} .{ }^{13} \mathrm{C} \mathrm{NMR}$ $\left(\mathrm{CDCl}_{3}, 75 \mathrm{MHz}\right), \delta: 213.1(\mathrm{C}), 170.2(\mathrm{C}), 71.7\left(\mathrm{CH}_{2}\right), 71.5\left(\mathrm{CH}_{2}\right), 71.0\left(\mathrm{CH}_{2}\right), 70.7\left(\mathrm{CH}_{2}\right), 68.3$ (C), $51.2(\mathrm{C}), 49.9\left(\mathrm{CH}_{2}\right), 48.3\left(\mathrm{CH}_{2}\right), 44.2\left(\mathrm{CH}_{2}\right), 43.5(\mathrm{CH}), 28.4\left(\mathrm{CH}_{2}\right), 27.5\left(\mathrm{CH}_{2}\right), 22.0$ $\left(\mathrm{CH}_{3}\right), 21.2\left(\mathrm{CH}_{3}\right)$ ppm. FTIR, v $\max : 1738$ (str), 1626 (str), $1119(\mathrm{~m}) \mathrm{cm}^{-1}$. MS (ESI) $\mathrm{m} / z(\%)$ : 362 (100), 340 (1). HRMS (FTMS-ESI) m/z: 340.2115 (Calcd. for $\mathrm{C}_{18} \mathrm{H}_{30} \mathrm{NO}_{5}$ : 340.2118). (1S,2R)-7,7-Dimethyl-1-[(1,4,7-trioxa-10-azacyclododecan-10-yl)carbonyl]norbornan-2-ol (7c). 9c (150 mg, $0.4 \mathrm{mmol})$ was reduced following the experimental procedure for the preparation of 7a. Purification was by column chromatography in hexanes/ethyl acetate (1/1).

7c. White solid, yield 99\%, $149 \mathrm{mg}, \mathrm{mp} 115-116{ }^{\circ} \mathrm{C}$. $[\alpha]_{\mathrm{D}}{ }^{20}-15.6\left(c\right.$ 0.390, $\left.\mathrm{CHCl}_{3}\right) .{ }^{1} \mathrm{H}$ NMR $\left(\mathrm{CDCl}_{3}, 300 \mathrm{MHz}\right), \delta: 4.74(\mathrm{~d}, J=4.8 \mathrm{~Hz}, 1 \mathrm{H}), 4.54-4.29(\mathrm{~m}, 1 \mathrm{H}), 4.21(\mathrm{dd}, J 4.3,4.3 \mathrm{~Hz}, 1 \mathrm{H})$, 4.11-3.94 (m, 1H), 3.74-3.44 (m, 12H), 3.02-2.62 (m, 2H), 2.02-1.90 (m, 2H), 1.84-1.73 (m, 2H), $1.59-1.51(\mathrm{~m}, 2 \mathrm{H}), 1.36(\mathrm{~s}, 3 \mathrm{H}), 1.26-1.11(\mathrm{~m}, 1 \mathrm{H}), 1.16(\mathrm{~s}, 3 \mathrm{H}) \mathrm{ppm} .{ }^{13} \mathrm{C} \mathrm{NMR}\left(\mathrm{CDCl}_{3}, 75\right.$ MHz), $\delta: 174.1(\mathrm{C}), 76.4(\mathrm{CH}), 69.0\left(\mathrm{CH}_{2}\right), 60.8(\mathrm{C}), 50.6(\mathrm{C}), 49.1\left(\mathrm{CH}_{2}\right), 44.7(\mathrm{CH}), 39.9$ $\left(\mathrm{CH}_{2}\right), 29.9\left(\mathrm{CH}_{2}\right), 27.5\left(\mathrm{CH}_{2}\right), 22.6\left(\mathrm{CH}_{3}\right), 21.0\left(\mathrm{CH}_{3}\right)$ ppm. FTIR, v max: 3457 (broad, d), 1627 (str), 1128 (m) cm $\mathrm{cm}^{-1}$. MS (ESI) $\mathrm{m} / z$ (\%): 364 (100), 342 (1). HRMS (FTMS-ESI) m/z: 342.2268 (calcd. for $\mathrm{C}_{18} \mathrm{H}_{32} \mathrm{NO}_{5}$ : 342.2275 ). 


\section{Typical procedure for the enantioselective ethylation of aldehydes}

Into a $10 \mathrm{~mL}$ flame-dried argon-purged round-bottom flask, equipped with a magnetic stirrer under argon, containing the ligand $(0.05 \mathrm{mmol})$ in dry hexane $(1 \mathrm{~mL})$, diethylzinc solution $(2.0$ mmol, 1.0 $\mathrm{M}$ in hexanes) was added at room temperature. The mixture was stirred at this temperature for $30 \mathrm{~min}$. Benzaldehyde $(1.0 \mathrm{mmol})$ was then added and the reaction mixture was stirred at room temperature for $5 \mathrm{~h}$. The reaction was quenched with $10 \% \mathrm{HCl}(3 \mathrm{~mL})$. The resulting mixture was extracted with $\mathrm{Et}_{2} \mathrm{O}(3 \times 3 \mathrm{~mL})$. The combined organic layers were filtered through Celite and the solvent evaporated. The obtained residue was dissolved in HPLC-grade hexanes and submitted to analysis by GC and chiral HPLC. 1-Phenylpropan-1-ol: Chiralpak IC, $260 \mathrm{~nm}, 2$-propanol/hexanes (2:98), $1.3 \mathrm{~mL} / \mathrm{min} . \mathrm{t}_{\mathrm{R}} 7.3 \mathrm{~min}(R), 7.8 \mathrm{~min}(S)$.

\section{Acknowledgements}

We thank MICINN (CTQ2010-18076) and UCM-Santander (GR35/10-A-910107) for supporting this work. E. M. S.-C. thanks UCM for a pre-doctoral grant.

\section{References and Notes}

1. Oguni, N.; Omi, T. Tetrahedron Lett. 1984, 25, 2823.

2. Noyori, R.; Kitamura, M. Angew. Chem. Int. Ed. Engl. 1991, 30, 49.

3. Soai, K.; Niwa, S. Chem. Rev. 1992, 92, 833.

4. Knochel, P.; Millot, N.; Rodrigues, A. L. Org. React. 2001, 58, 417.

5. $\mathrm{Pu}, \mathrm{L} . ; \mathrm{Yu}, \mathrm{H}$. -B. Chem. Rev. 2001, 101, 757.

6. Pu, L. Tetrahedron 2003, 59, 9873.

7. Denmark, S. E.; Fu, J. Chem. Rev. 2003, 103, 2763.

8. Zhu, H.-J.; Jiang, J.-X.; Ren, J.; Yan, Y.-M.; Pitmann, C. U. Curr. Org. Synth. 2005, 2, 547.

9. Ramón, D. J.; Yus, M. Chem. Rev. 2006, 106, 2126.

10. Hatano, M.; Miyamoto, T.; Ishihara, K. Curr. Org. Chem. 2007, 11, 127.

11. Trost, B.; Weiss, A. H. Adv. Synth. Catal. 2009, 351, 963.

12. Somanathan, R.; Flores-López, L. Z.; Montalbo-González, R.; Chavez, D.; Parra-Hake, M.; Aguirre, G. Mini-Rev. Org. Chem. 2010, 7, 10.

13. Sedelmeir, J. Carsten B. J. Org. Chem. 2007, 72, 8859 and references cited therein.

14. Yang, Y.; Zhu, S.-F.; Zhou, C.-Y.; Zhou, Q.-L. J. Am. Chem. Soc. 2008, 130, 14052 and references cited therein.

15. Armstrong, J. D.; McWilliams, J. C. U.S. Patent 5977371.

16. Kitamura, M.; Suga, S.; Kawai, K.; Noyori, R. J. Am. Chem. Soc. 1986, 108, 6071.

17. Rodríguez-Escrich, S; Reddy, K. S.; Jimeno, C.; Colet, G.; Rodríguez-Escrich, C.; Solà, L.; Vidal-Ferrán, A.; Pericàs, M. A. J. Org. Chem. 2008, 73, 5340. 
18. (a) Wang, M.-C.; Zhang, Q.-J.; Wang, X.-D.; Ding, X.; Jing, T.-T.; Song, M.-P. J. Org. Chem. 2008, 73, 168. (b) Wang, M.-C.; Zhang, Q.-J.; Li, G.-W.; Liu, Z.-K. Tetrahedron: Asymmetry 2009, 20, 288.

19. Márquez Sánchez-Carnerero, E.; de las Casas Engel, T.; Lora Maroto, B.; de la Moya Cerero, S. Tetrahedron: Asymmetry 2009, 20, 2655.

20. de las Casas Engel, T.; Lora Maroto, B.; García Martínez, A.; de la Moya Cerero, S. Tetrahedron: Asymmetry 2008, 19, 646.

21. de las Casas Engel, T.; Lora Maroto, B.; de la Moya Cerero, S. Eur. J. Org. Chem. 2010, 9, 1717.

22. Intermediate 9a had been prepared before from ketopinic acid chloride: Hari, Y.; Aoyama, T. Synthesis 2005, 583.

23. $\mathrm{dr}>98$ / <2 (exo/endo) by NMR. Each exo diastereomer could be obtained pure after column chromatography. 